Supporting Information

\title{
Torsional Constraints of DNA Substrates Impact Cas9 Cleavage
}

Michael H. Räz, ${ }^{\dagger}$ Kumi Hidaka, ${ }^{\S}$ Shana J. Sturla, ${ }^{\dagger}$ Hiroshi Sugiyama, ${ }^{*, \$}$ and Masayuki Endo,,‡

Institute for Integrated Cell-Material Sciences (WPI-iCeMS), Kyoto University, Yoshidaushinomiyacho, Sakyo-ku, Kyoto 606-8501, Japan

${ }^{\S}$ Department of Chemistry, Graduate School of Science, Kyoto University, Kitashirakawa-oiwakecho, Sakyo-ku, Kyoto 606-8502, Japan

†Department of Health Sciences and Technology, ETH Zürich, Schmelzbergstrasse 9, 8092 Zürich, Switzerland

${ }^{*}$ To whom the correspondence should be addressed:

E-mail: endo@kuchem.kyoto-u.ac.jp,hs@kuchem.kyoto-u.ac.jp 
(A)

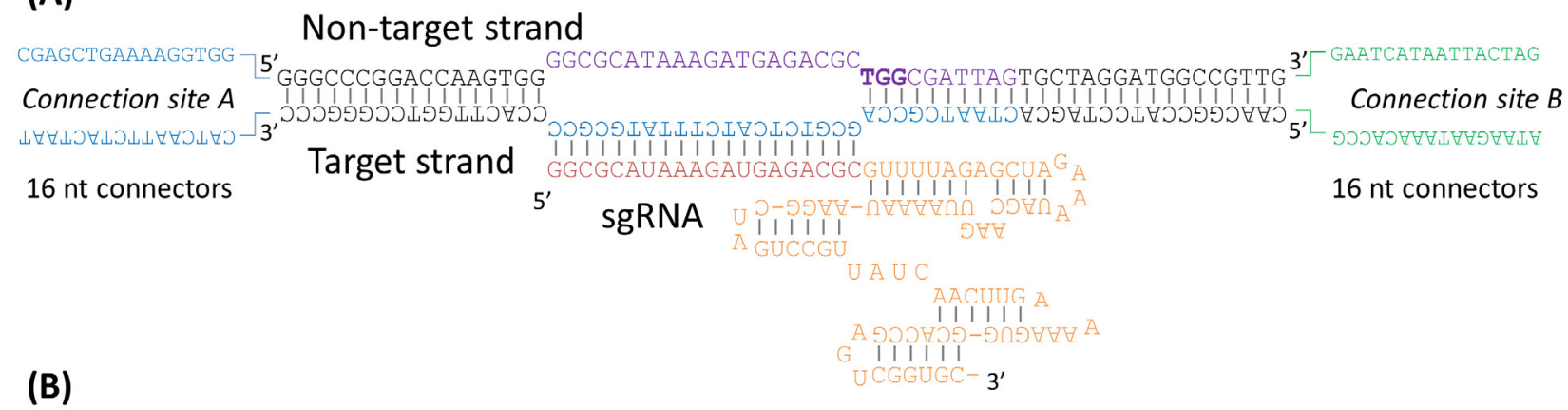

I: Both strands constrained

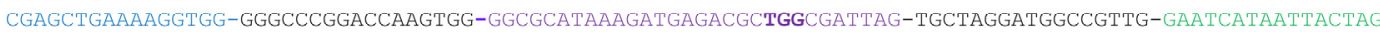

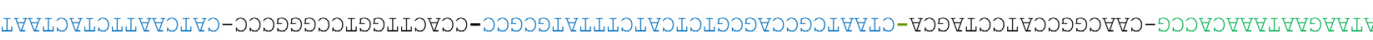

II: Target strand constrained

CGAGCTGAAAAGGTG-GGGCCCGGACCAAGTGG-GGCGCATAAAGATGAGACGCTGGCGATTAG-TGCTAGGATGGCCGTTG-GAATCATAATTACTAG

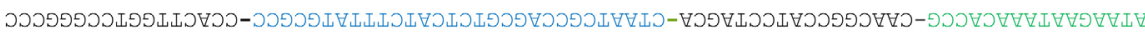

III: Non-target strand constrained

CGAGCTGAAAAGGTGG-GGGCCCGGACCAAGTGG-GGCGCATAAAGATGAGACGCTGGCGATTAG-TGCTAGGATGGCCGTTG

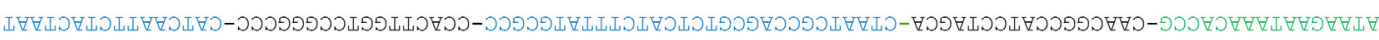

IV: Both strands rotatable

CGAGCTGAAAAGGTGG-GGGCCCGGACCAAGTGG-GGCGCATAAAGATGAGACGCTGGCGATTAG-TGCTAGGATGGCCGTTG

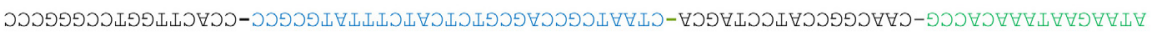

(C)

$64 \mathrm{bp}$

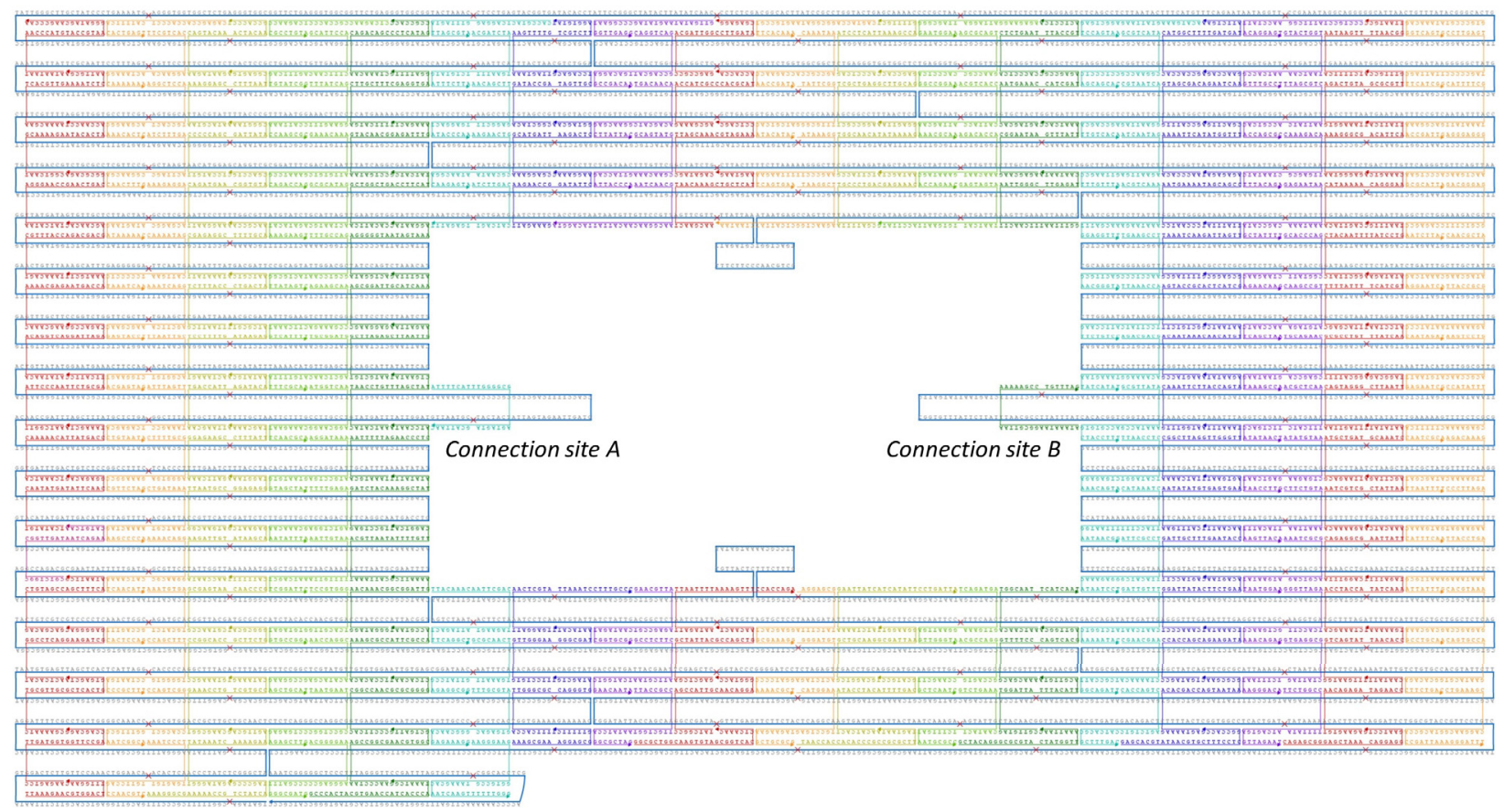

Figure S1. DNA and RNA sequences used for the experiments and DNA frame structure. (A) dsDNA and sgRNA sequences for complex formation. (B) Four substrate dsDNA with different 16nt connectors. (C) DNA frame structure for incorporation of substrate dsDNAs. ${ }^{[1]}$ 


\section{Materials and Methods}

sgRNA preparation: In order to prepare the single guide RNA (sgRNA) we prepared a T7 promoter containing dsDNA template for in vitro transcription by performing overlap extension PCR with an 84nt and 56nt template strand (Table S1). The PCR amplification was performed using Go Taq polymerase (Promega) with a forward and reverse primer (Table S1) by following the manufactures protocol. The amplified product was purified using a QIAquick PCR purification Kit (Qiagen).

The T7 promoter containing dsDNA was further used for the in vitro transcription of the sgRNA. The reaction was performed using $30 \mathrm{nM}$ dsDNA template, $40 \mathrm{mM}$ Tris- $\mathrm{HCl}(\mathrm{pH} 8.0), 5 \mathrm{mM}$ DTT, $8 \mathrm{mM}$ $\mathrm{MgCl}_{2}, 2 \mathrm{mM}$ spermidine, $2 \mathrm{mM}$ NTP and $0.25 \mu \mathrm{M}$ T7 RNA polymerase (Takara) at $37{ }^{\circ} \mathrm{C}$ for $2 \mathrm{~h}$. The sgRNA was purified by polyacrylamide gel electrophoresis and the quality was confirmed by running the sgRNA on an $8 \%$ denaturing polyacrylamide gel (Figure S2).

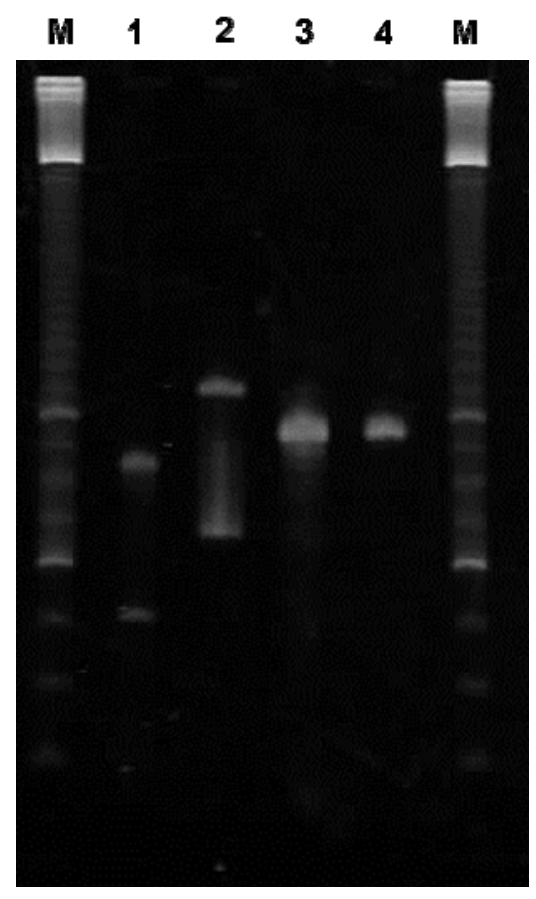

Figure S2. Analysis of sgRNA preparation on $8 \mathrm{M}$ Urea / 12\% polyacrylamide gel, stained with SYBR gold. M: 10 bp marker, lane 1: $56 \mathrm{nt}$ and $84 \mathrm{nt}$ templates for the overlap extension PCR; Lane 2: PCR product, 116 bp; Lane 3: sgRNA, 96 nt, before purification; Lane 4: sgRNA, 96 nt, after purification. 
Table S1. DNA sequences and PCR primes for in vitro transcription of sgRNA.

Sequences for in vitro transcription of sgRNA (5' - 3') 1

84 nt T7 promoter +

TTCTAATACGACTCACTATAGGCGCATAAAGATGAGACGCGTTT

sgRNA template top

TAGAGCTAGAAATAGCAAGTTAAAATAAGGCTAGTCCGTT

2

56nt $\mathrm{T} 7$ promoter +

GCACCGACTCGGTGCCACTTTTTCAAGTTGATAACGGACTAGC

sgRNA template bottom

CTTATTTTAACTT

PCR primers (5' - 3')

3 22nt forward primer

TTCTAATACGACTCACTATAGG

422 nt reverse primer

GCACCGACTCGGTGCCACTTTT

DNA substrate strands: Self-assembly of the DNA origami frame with the dsDNA substrate: The four different dsDNA substrates (I-IV) were prepared by combining the 96mer and 80mer ssDNA sequences (Figure 1) heating to $95{ }^{\circ} \mathrm{C}$ and cool to room temperature. The DNA origami frame containing the dsDNA substrate was obtained by annealing $10 \mathrm{nM}$ of M13mp18 single stranded DNA (New England BioLabs, Ipswich, MA) with $50 \mathrm{nM}$ staple strands (226 strands) and $100 \mathrm{nM}$ of the pre mixed dsDNA substrate strands (I, II, III or IV) from $80{ }^{\circ} \mathrm{C}$ to $15{ }^{\circ} \mathrm{C}$ at a rate of $-1.0{ }^{\circ} \mathrm{C} \mathrm{min}{ }^{-1}$. The annealing reaction was carried out in a buffer containing $20 \mathrm{mM}$ Tris-HCl (pH 7.6), $1 \mathrm{mM}$ EDTA and 10 $\mathrm{mM} \mathrm{MgCl}_{2}$. Subsequently, the assembled DNA origami frames were purified by gel-filtration chromatography (GE sephacryl-300, GE Healthcare Japan, Tokyo, Japan). The incorporated substrate sequences and listed in the Supporting Information.

Observation of Cas9 complex formation with the dsDNA substrate in the DNA origami frame: AFM imaging of streptococcus pyogenes Cas9 complex formation was achieved by pre-incubation of 10 $\mathrm{nM}$ of Cas 9 with $10 \mathrm{nM}$ sgRNA at $25{ }^{\circ} \mathrm{C}$ for $10 \mathrm{~min}$ in a reaction buffer containing $10 \mathrm{mM}$ Tris- $\mathrm{HCl}$ (pH 7.6), $10 \mathrm{mM} \mathrm{NaCl}, 10 \mathrm{mM} \mathrm{MgCl} 2$ and $0.1 \mathrm{mM}$ EDTA. Subsequently, $1 \mathrm{nM}$ of the dsDNA substrate containing DNA origami frame was added to the Cas9/sgRNA complex and the reaction was kept at $37^{\circ} \mathrm{C}$. At well-defined time-points $2 \mu \mathrm{L}$ of the reaction mixture were removed, and immediately placed on freshly cleaved mica disc. After 2 - 20 minutes of incubation the sample was rinsed with $10 \mu \mathrm{L}$ of the aforementioned reaction buffer and imaged in the same buffer. AFM imaging was performed on a highspeed AFM (Nano-Live Vision; RIBM, Tsukuba, Japan) using a silicon nitride cantilever (Olympus BLAC10EGS). 
Quantification of Cas9 substrate cleavage in the DNA origami frame by Real-Time PCR: The dsDNA substrate containing DNA origami frame was prepared as described under self-assembly of the DNA origami frame but instead of gel-filtration chromatography the assembled frames were purified by $1 \%$ agarose gel electrophoresis. Subsequently, the corresponding bands were cut out and recovered using Freeze 'N Squeeze DNA gel extraction columns (BioRad) following the manufactures protocol.

The Cas9 cleavage reaction (Cas9 +) was performed in $20 \mu \mathrm{L}$ reaction containing $5 \mathrm{nM}$ of the preincubated Cas9/sgRNA complex $\left(25^{\circ} \mathrm{C}\right.$ for $\left.10 \mathrm{~min}\right), 10 \mathrm{mM}$ Tris- $\mathrm{HCl}(\mathrm{pH} 7.6), 10 \mathrm{mM} \mathrm{NaCl}, 10 \mathrm{mM}$ $\mathrm{MgCl}_{2}, 0.1 \mathrm{mM}$ EDTA and $1 / 4$ of the gel-purified DNA origami frame with the corresponding dsDNA substrate (I-IV). The reference reaction (Cas9 -) was prepared in exact the same way as described for the Cas9 + reaction, but the pre-incubation step contained only sgRNA and no Cas9. Both reactions (Cas9+ and Cas9 -) were kept at $37^{\circ} \mathrm{C}$ and after $60 \mathrm{~min}$ and $240 \min 5 \mu \mathrm{L}$ were removed, transferred into a new tube, heat inactivated and used for the qPCR reaction.

Real-time PCR reactions were performed with a Thermal Cycler Dice ${ }^{\circledR}$ Real Time System III (Takara) and the subsequent calculations were carried out with the corresponding software LCD Software version 1.00. The PCR reactions contained 1 x SYBR ${ }^{\circledR}$ Fast qPCR Mix (Takara/Clontech), $400 \mathrm{nM}$ forward and reverse primers and the $5 \mu \mathrm{L}$ of the DNA substrate containing DNA origami frame, after the Cas $9+$ or Cas 9 - reaction, in a total volume of $20 \mu \mathrm{L}$. The PCR reaction contained of 1 initial denaturation step at $95^{\circ} \mathrm{C}$ for $30 \mathrm{~s}$, followed by 40 cycles containing of a denaturation at $95{ }^{\circ} \mathrm{C}$ for $5 \mathrm{~s}$ and a hybridization/amplification step at $60{ }^{\circ} \mathrm{C}$ for $20 \mathrm{~s}$. The standard curve was obtained using 500, 100, 20, 4 and 0.8 pM DNA templates. The dsDNA substrate and primer sequences are listed in Table S2. 
Table S2. DNA sequences for the four different substrates and qPCR primer sequences.

Substrate strands $\left(5^{\prime}-3^{\prime}\right)$

$196 n t$ top strand

CGAGCTGAAAAGGTGGGGGCCCGGACCAAGTGGGGCGCATAAAG ATGAGACGCTGGCGATTAGTGCTAGGATGGCCGTTGGAATCATAAT $\underline{\text { TACTAG }}$

ATAAGAATAAACACCGCAACGGCCATCCTAGCACTAATCGCCAGCG

2 96nt bottom strand TCTCATCTTTATGCGCCCCACTTGGTCCGGGCCCCATCAATTCTACT $\underline{\mathrm{AAT}}$

3 80nt top strand

CGAGCTGAAAAGGTGGGGGCCCGGACCAAGTGGGGCGCATAAAG ATGAGACGCTGGCGATTAGTGCTAGGATGGCCGTTG

4 80nt bottom strand ATAAGAATAAACACCGCAACGGCCATCCTAGCACTAATCGCCAGCG TCTCATCTTTATGCGCCCCACTTGGTCCGGGCCC

qPCR primers (5' - 3')

5 18nt forward primer GGGCCCGGACCAAGTGGG

6 18nt reverse primer CAACGGCCATCCTAGCAC

Straight underlined sequences represent complementary sequences to the connection sites in the DNA frame. Wavelike underlined sequences represent complementary sequences for the forward or reverse primer. Nucleotides in bold represent the PAM site.

(A)

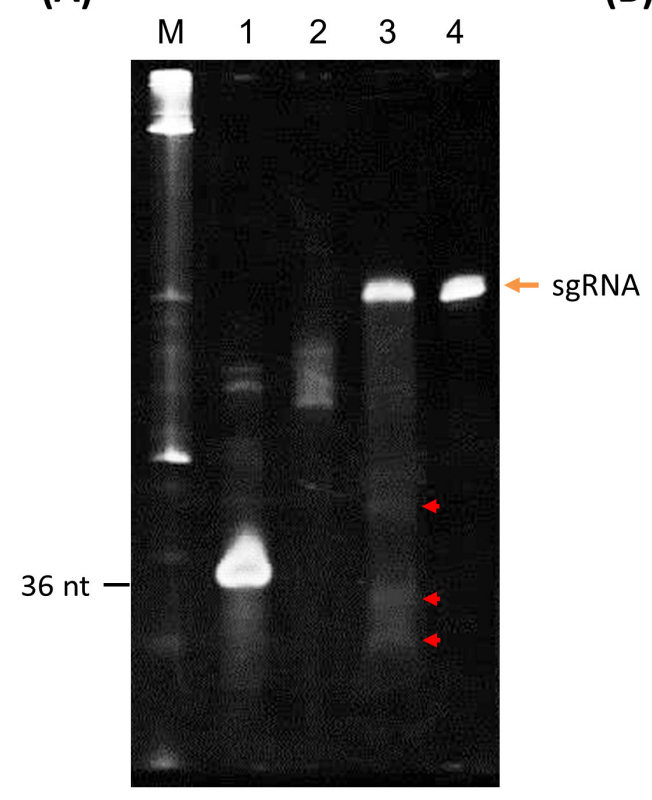

(B)

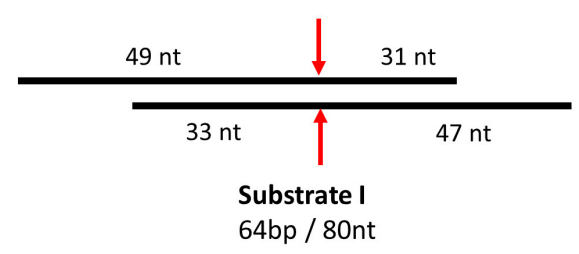

Figure S3. Analysis of Cas9 substrate cleavage on $8 \mathrm{M}$ Urea / 12\% polyacrylamide gel, stained with SYBR gold. M: 10 bp marker; Lane 1: 36nt marker; Lane 2: 64 bp/80nt DNA substrate; Lane 3: Cas9, sgRNA + 64 bp/80nt DNA substrate (5:5:1). Lane 4: sgRNA. Red arrows indicate the expected cleavage products. Orange arrow indicates the sgRNA. Expected product length after cleavage of substrate I (64 bp/80nt). Red arrows indicate cleavage site. 

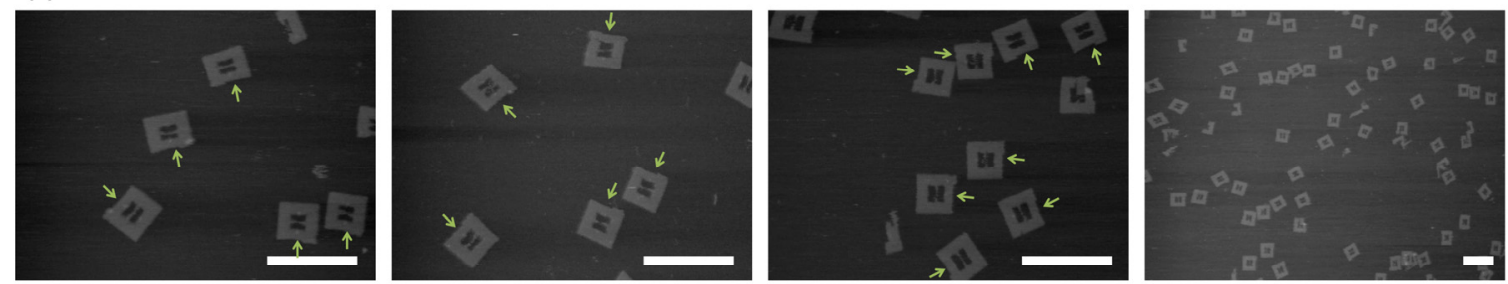

(b) $15 \mathrm{~min}$
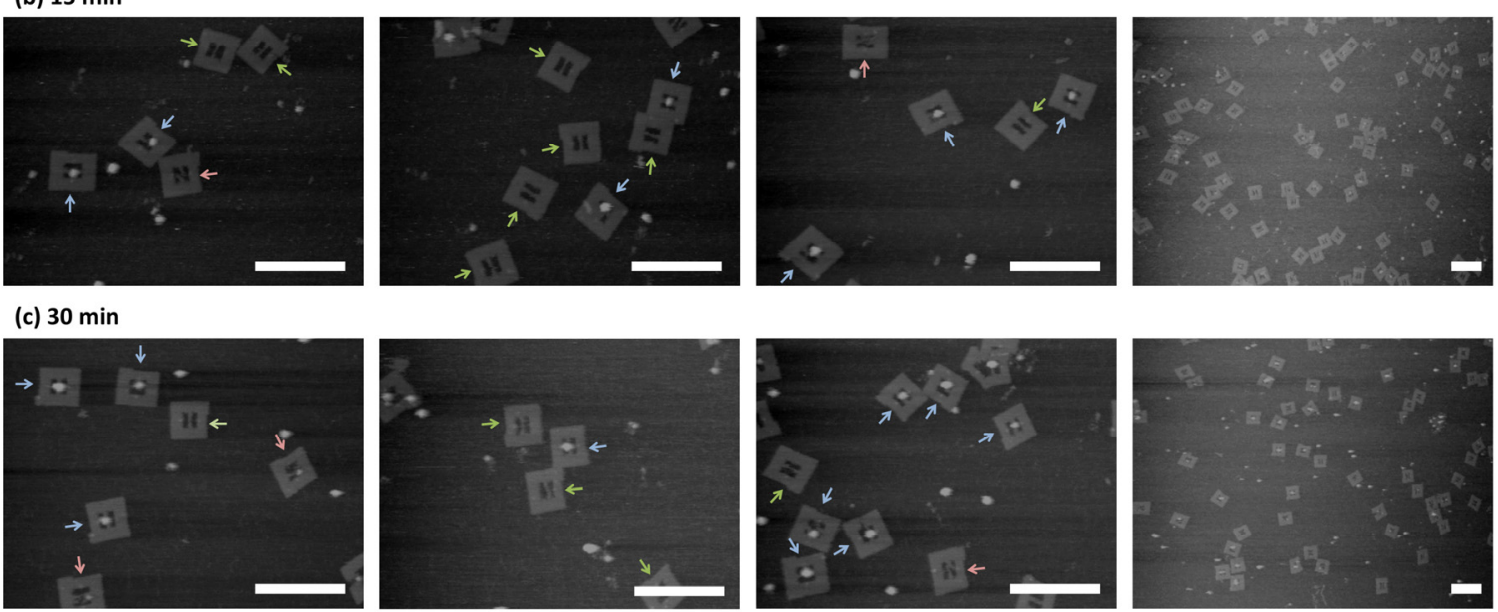

(d) $60 \mathrm{~min}$
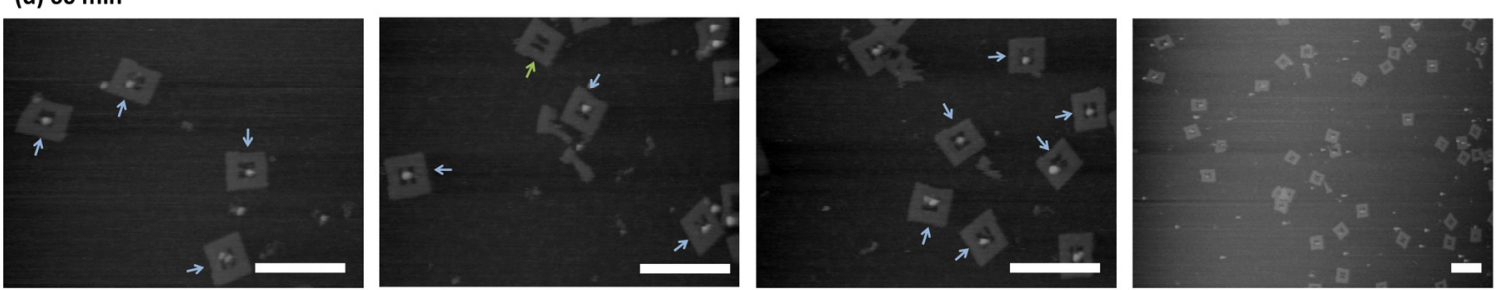

(e) $90 \mathrm{~min}$
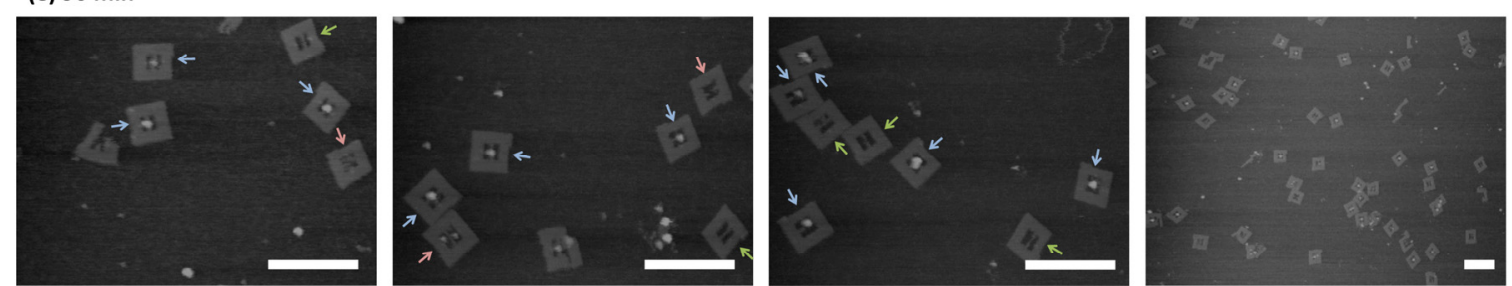

(f) $240 \mathrm{~min}$
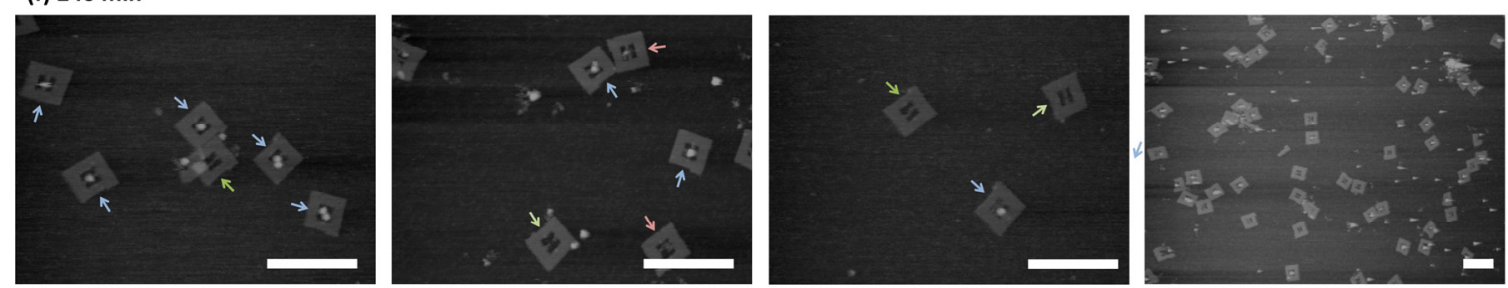

(f) $20 \mathrm{~h}$
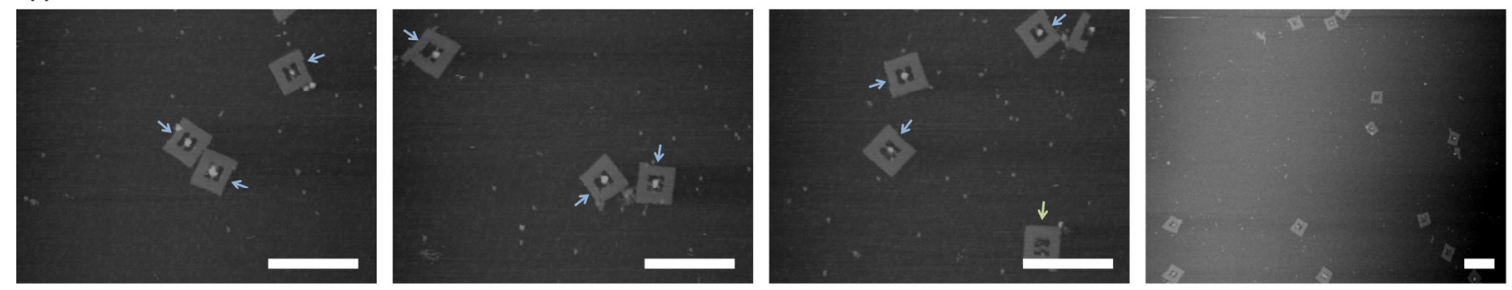

Figure S4. AFM images of DNA frame containing substrate I incubation with Cas9/sgRNA. Scale bar $200 \mathrm{~nm}$. 

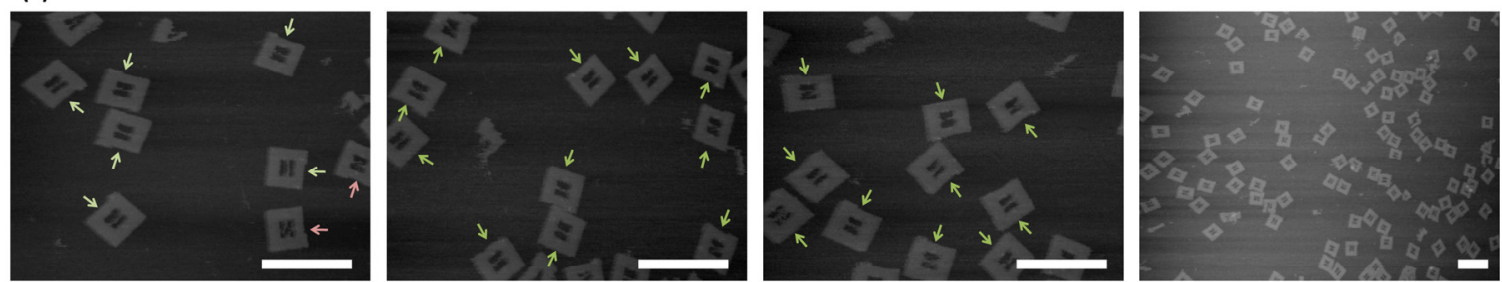

\section{(b) $15 \mathrm{~min}$}
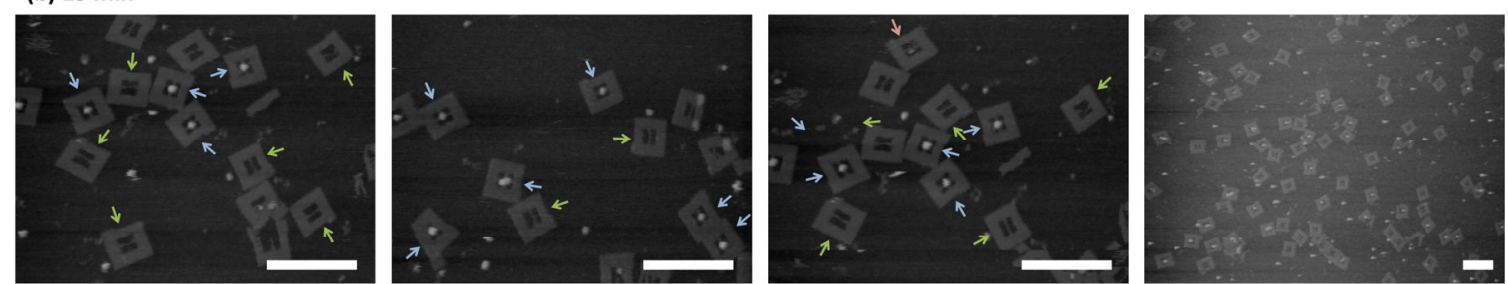

(c) $30 \mathrm{~min}$
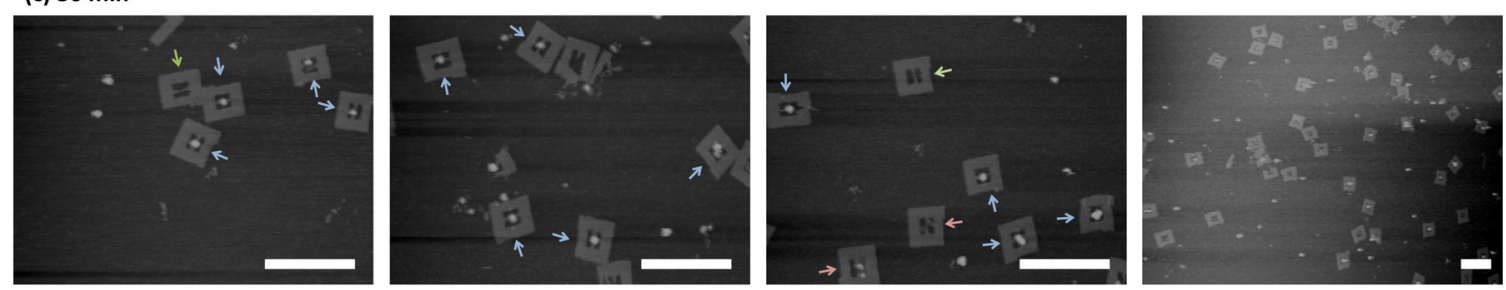

(d) $60 \mathrm{~min}$
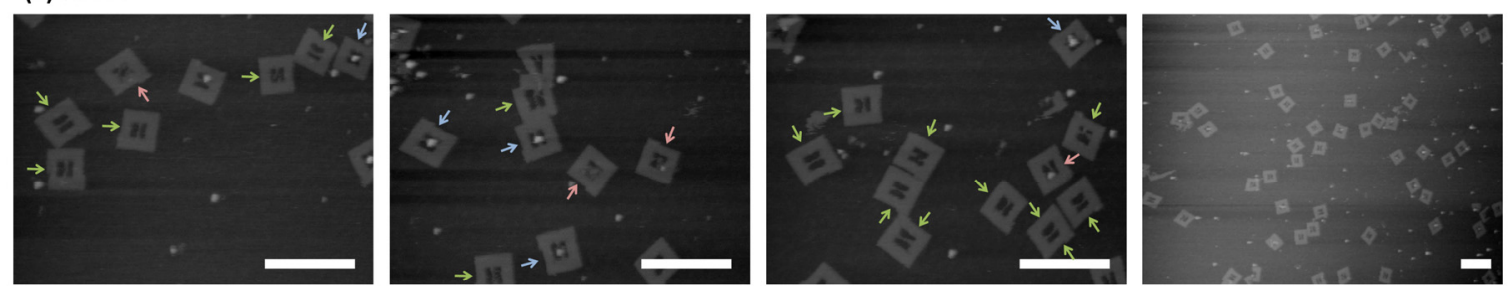

(e) $90 \mathrm{~min}$
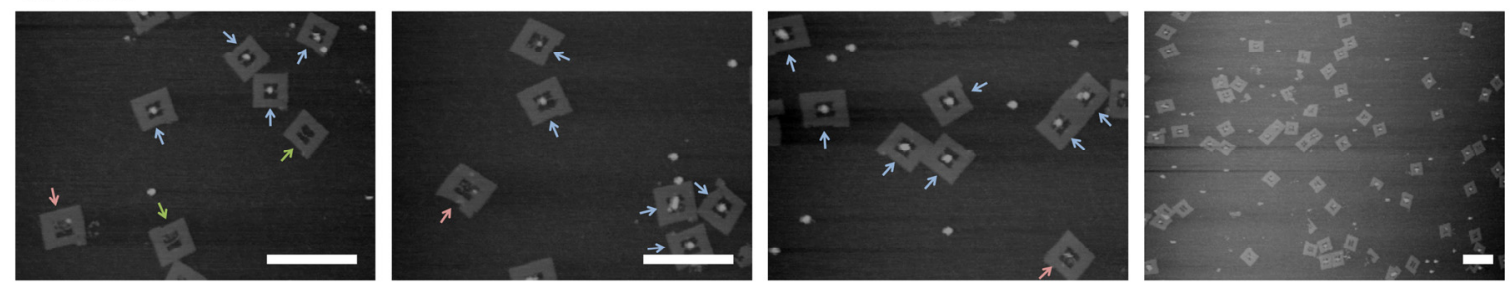

(f) $240 \mathrm{~min}$
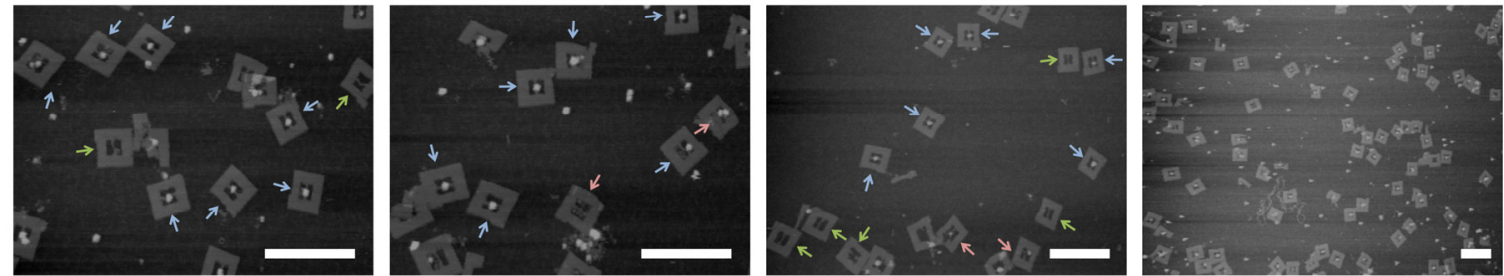

(f) $20 \mathrm{~h}$
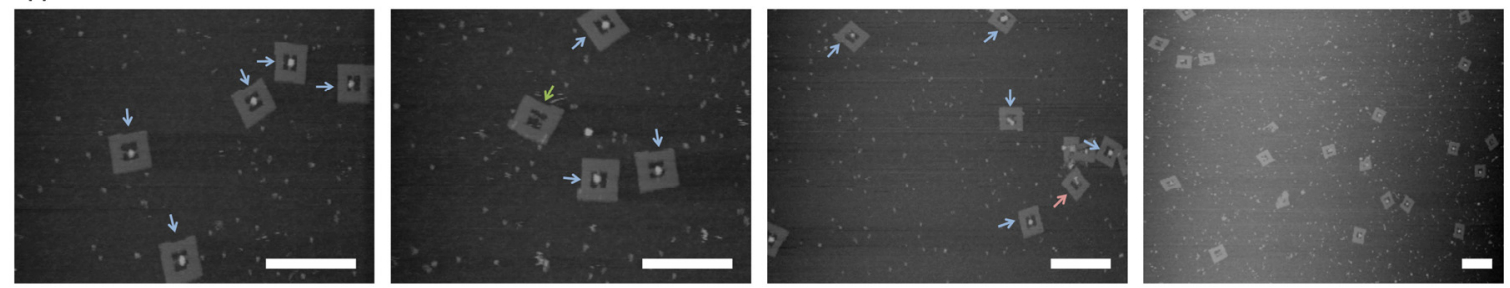

Figure S5. AFM images of DNA frame containing substrate II incubation with Cas9/sgRNA. Scale bar $200 \mathrm{~nm}$. 

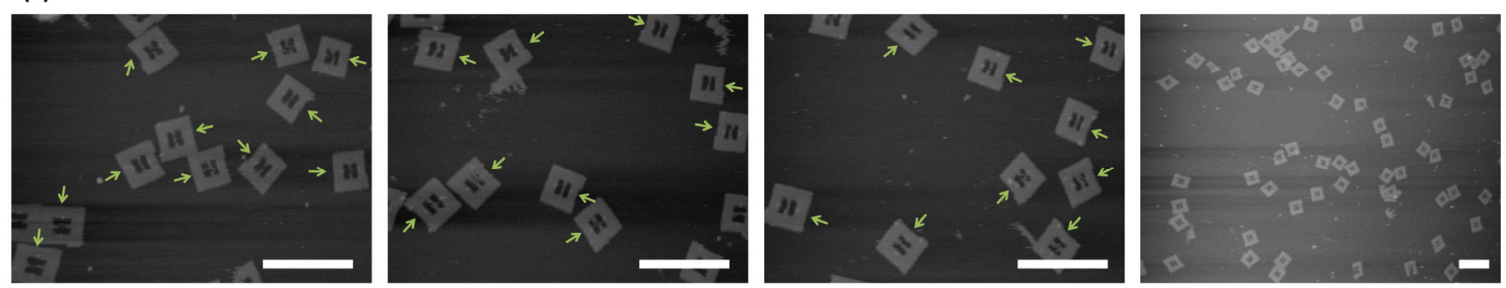

\section{(b) $15 \mathrm{~min}$}
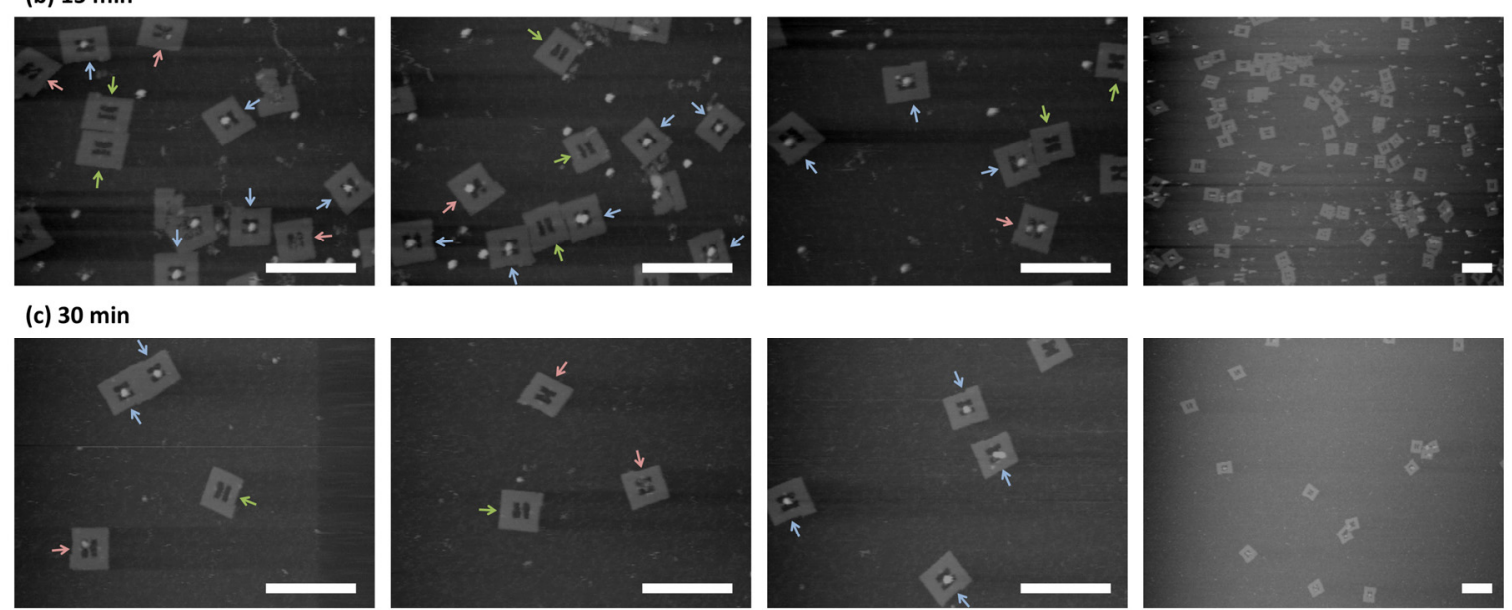

(d) $60 \mathrm{~min}$
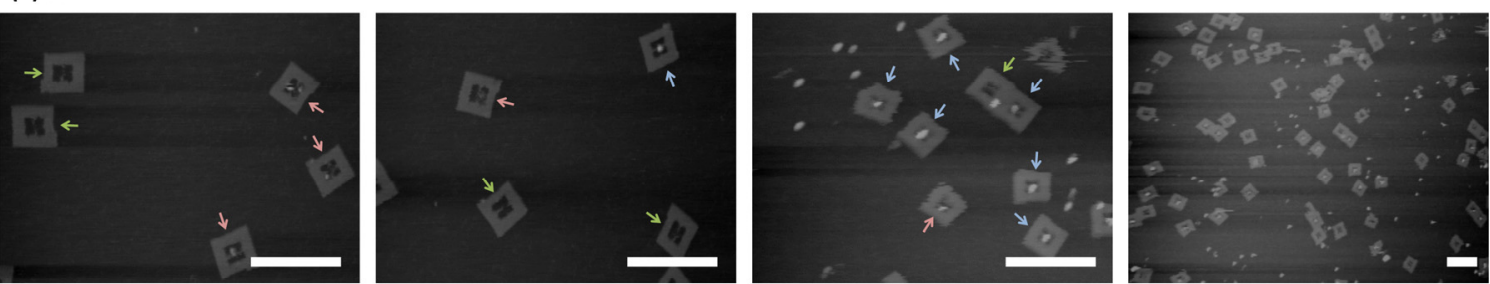

(e) $90 \mathrm{~min}$
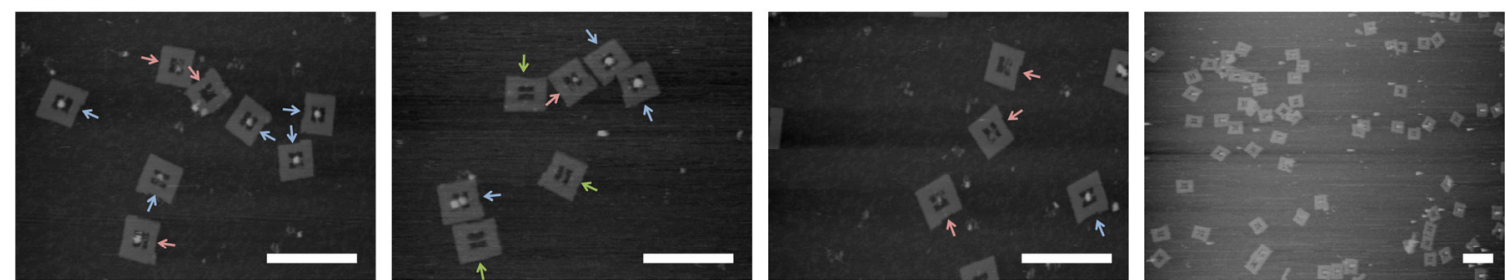

(f) $240 \mathrm{~min}$
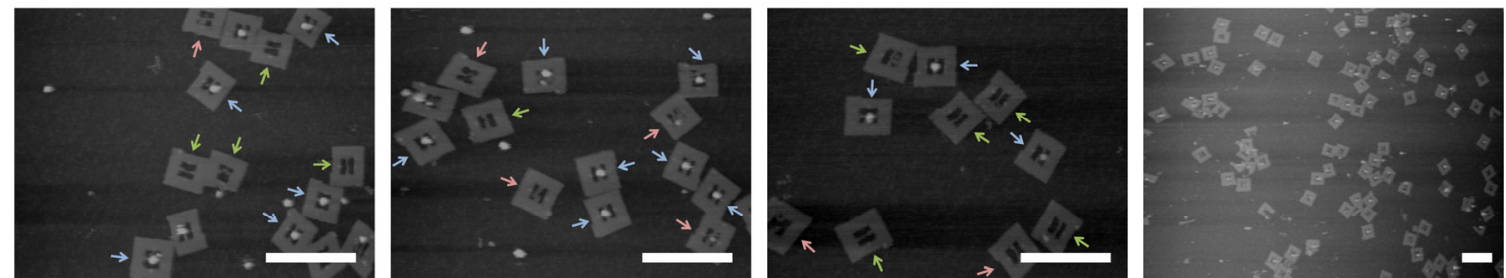

(f) $20 \mathrm{~h}$
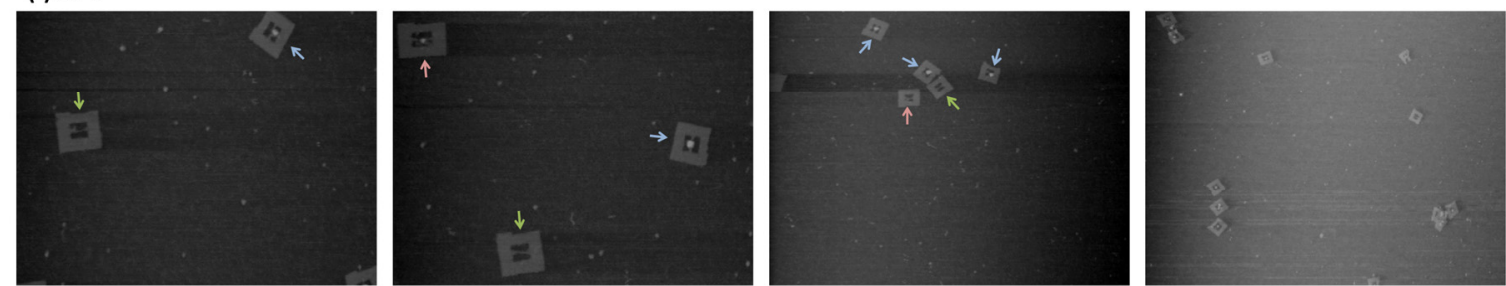

Figure S6. AFM images of DNA frame containing substrate III incubation with Cas9/sgRNA. Scale bar $200 \mathrm{~nm}$. 

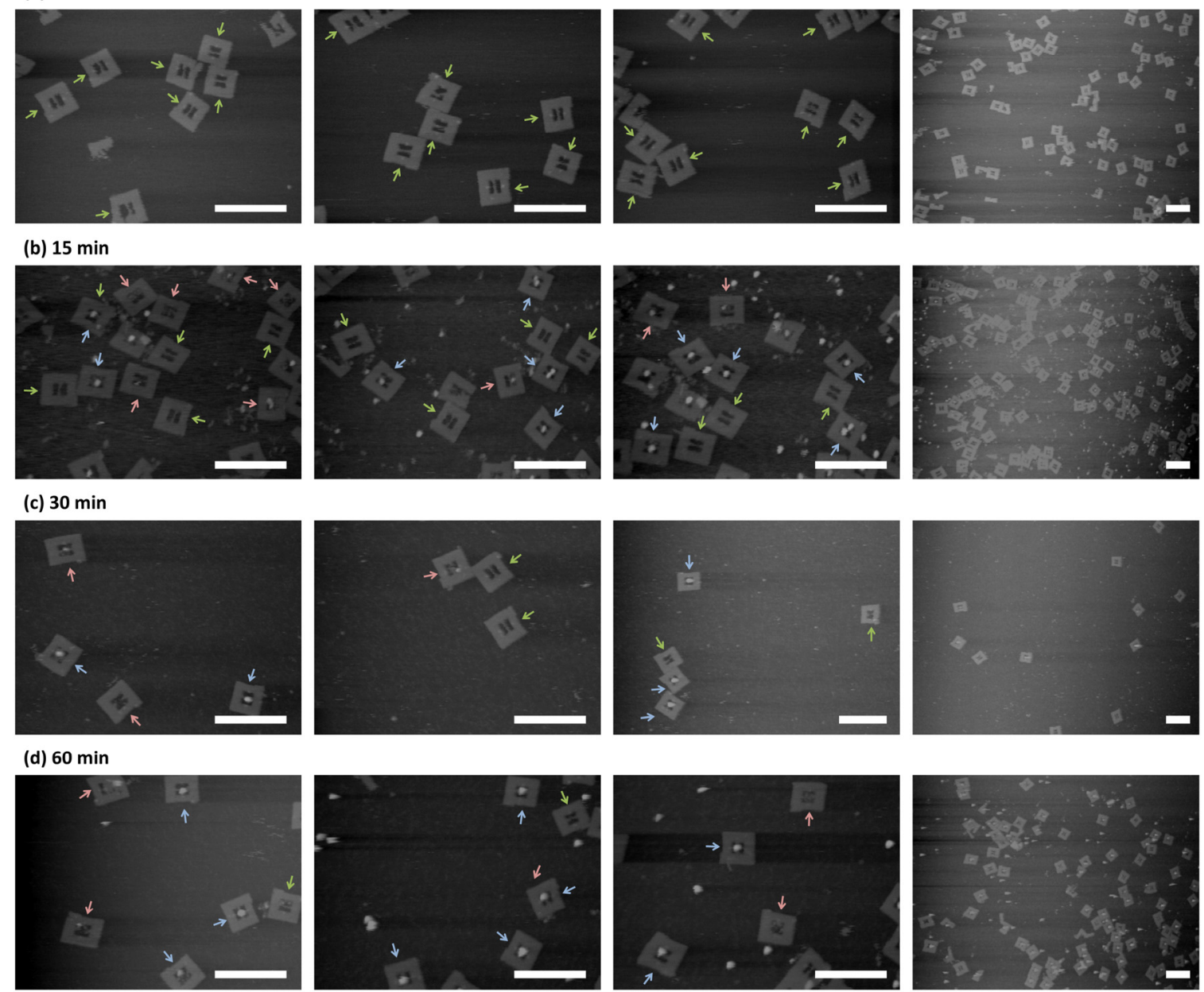

(e) $90 \mathrm{~min}$
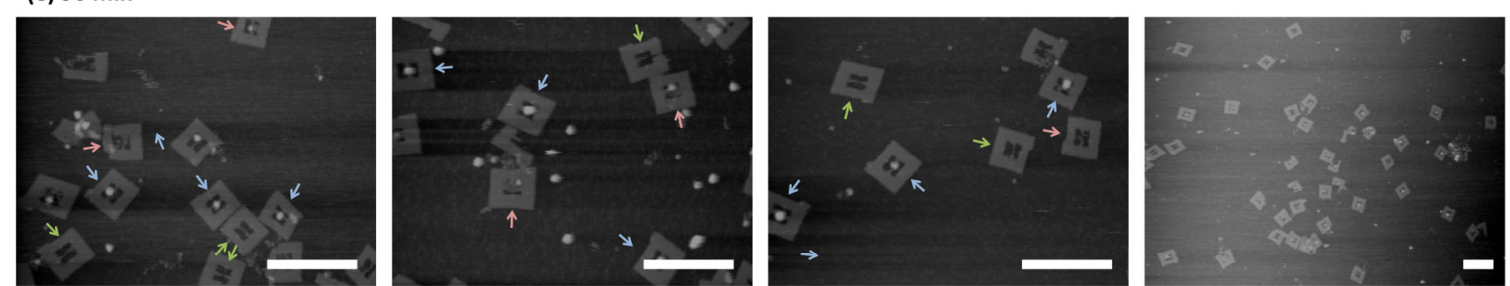

(f) $240 \mathrm{~min}$
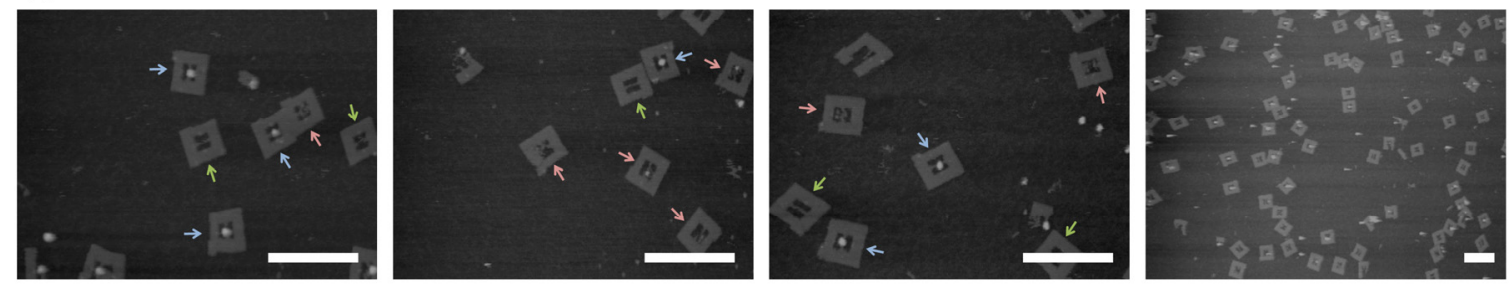

(f) $20 \mathrm{~h}$
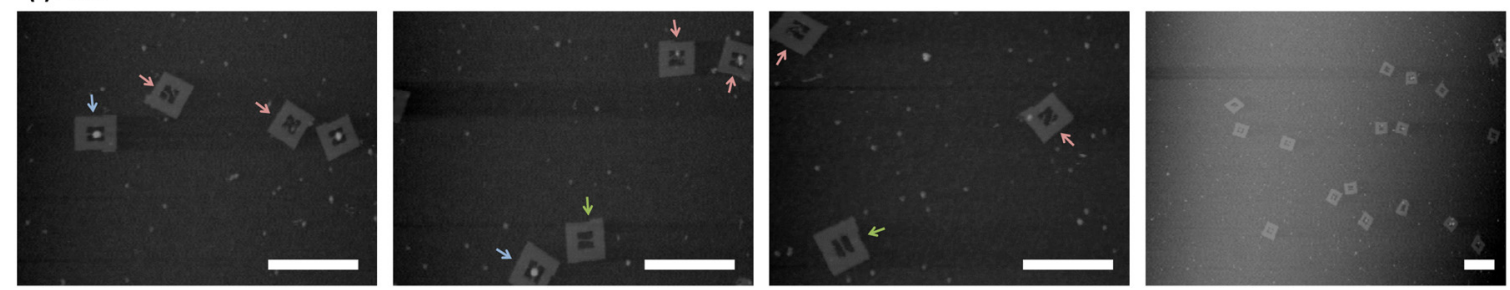

Figure S7. AFM images of DNA frame containing substrate IV incubation with Cas9/sgRNA. Scale bar $200 \mathrm{~nm}$. 


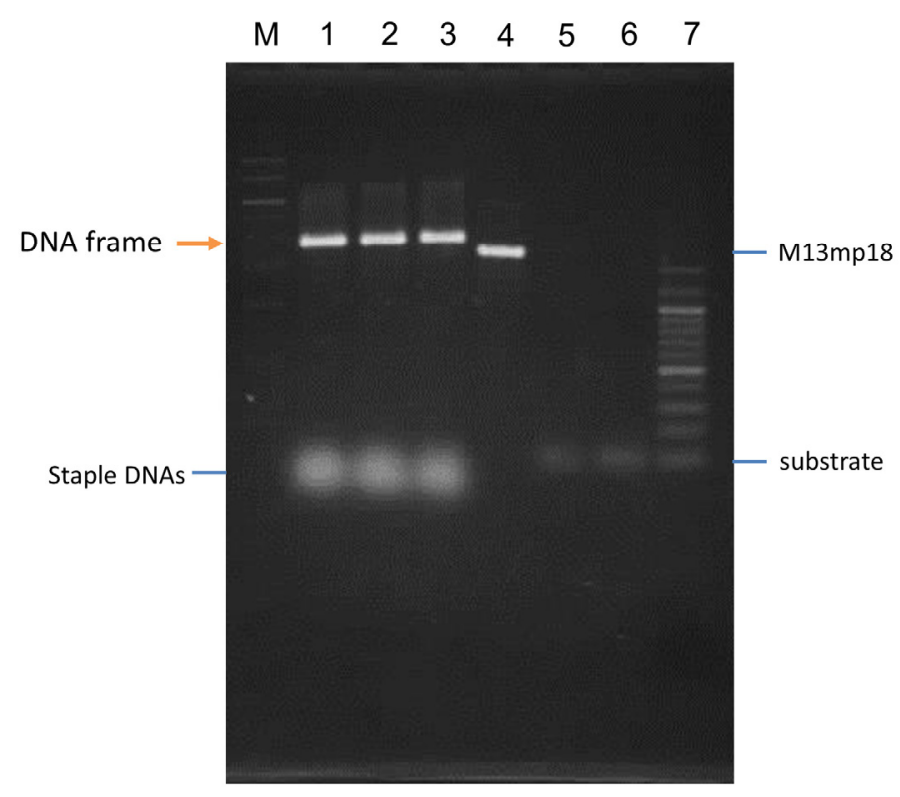

Figure S8. $1 \%$ agarose gel purification of folded DNA origami frames, stained with EtBr. M: $1 \mathrm{~kb}$ marker; Lane 1: DNA origami frame without substrate; Lane 2: DNA origami frame with 64mer substrate; Lane 3: DNA origami frame with 74mer substrate; Lane 4: M13mp18; Lane 5: 64mer substrate; Lane 6: 74mer substrate; Lane 7: 100 bp marker. Red arrow indicates the unbound staple strands.
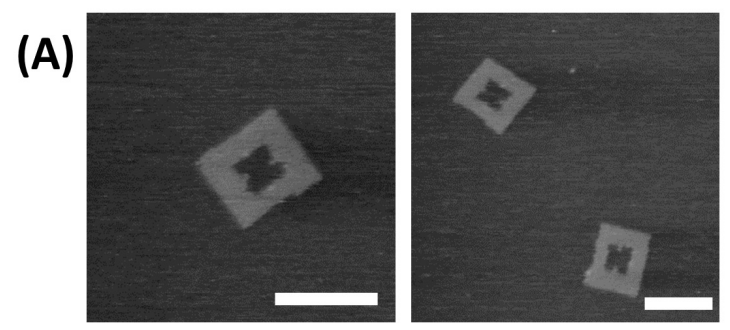

(B)
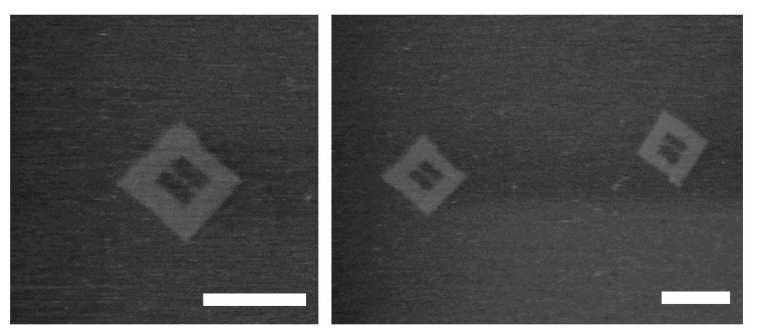

Figure S9. AFM check of the DNA origami frames after agarose gel purification. (A) DNA origami frame without substrate. (B) DNA origami frame containing 64mer substrate. Scale bar $100 \mathrm{~nm}$. 
Table S3. Amplification curves of qPCR reactions with substrate I, II, III or IV containing Frames after 60 min Cas9 reactions.
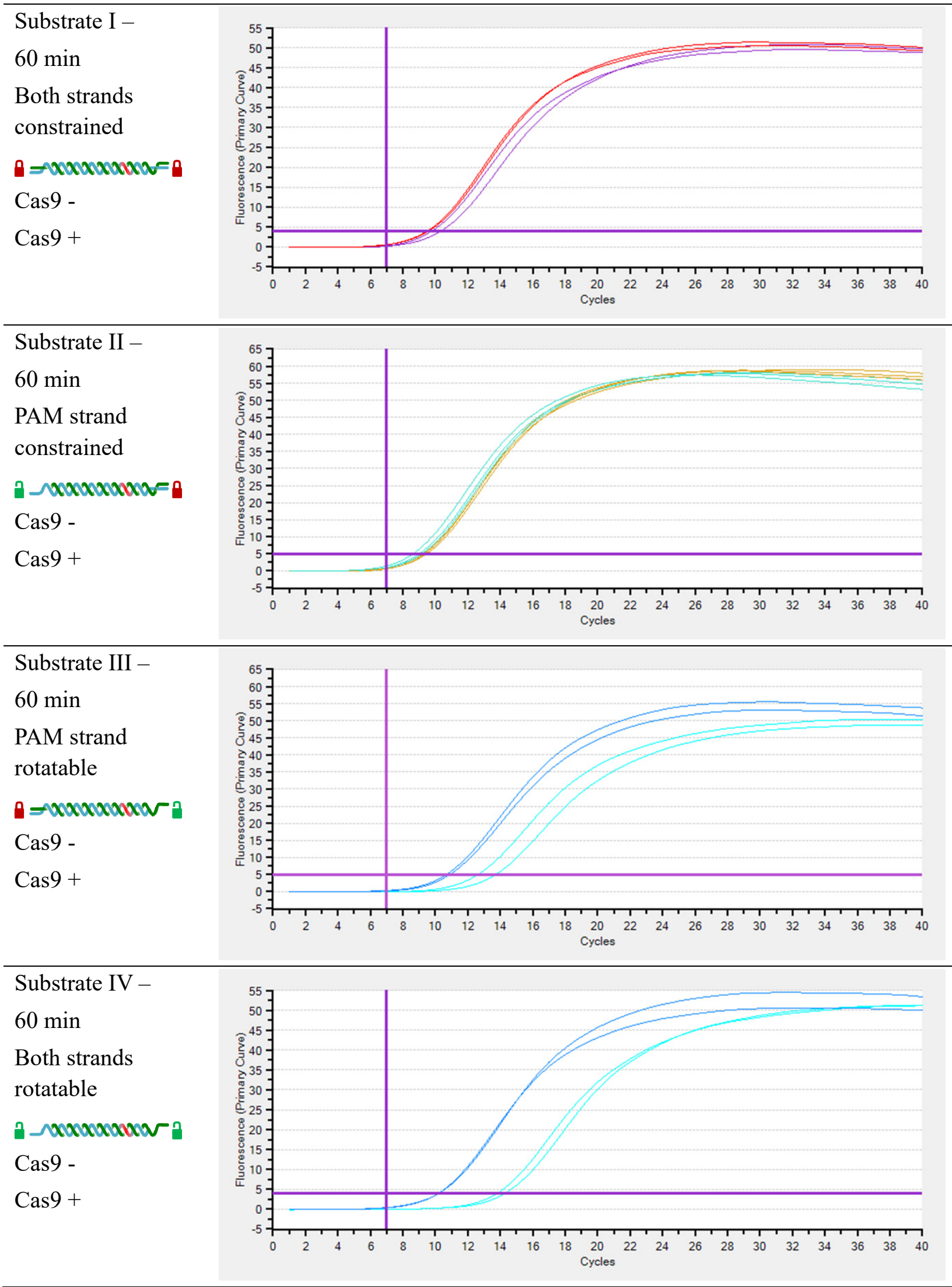
Table S4. Amplification curves of qPCR reactions with substrate I, II, III or IV containing Frames after 240 min Cas9 reactions.

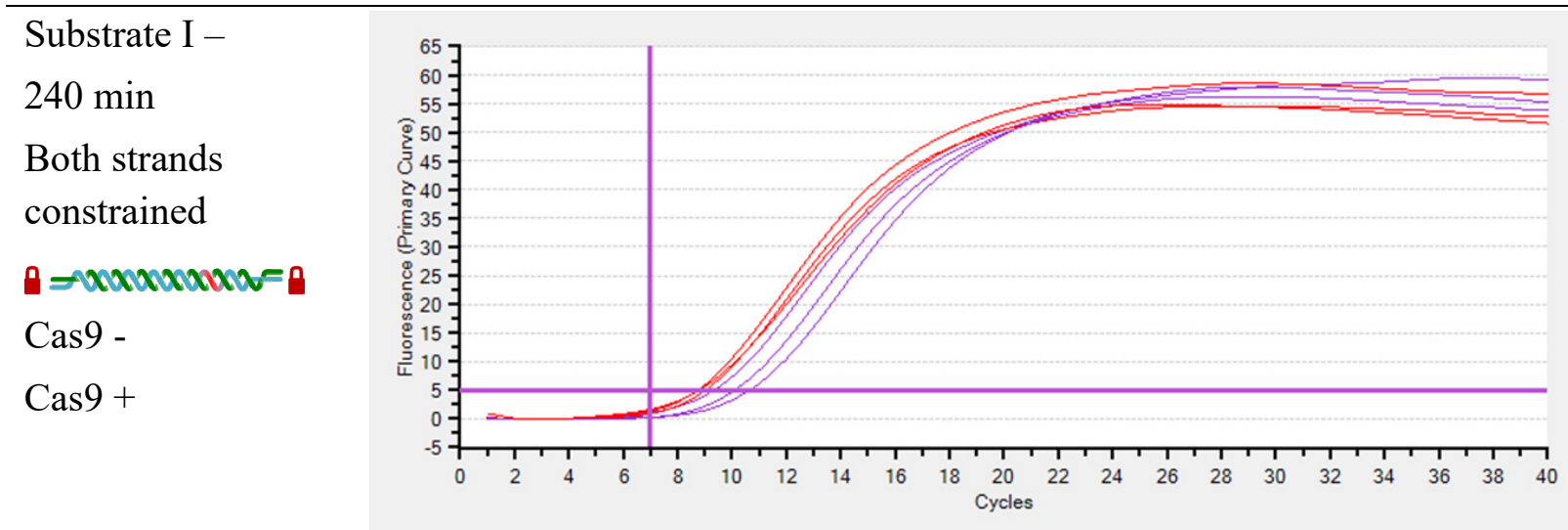

Substrate II -

$240 \mathrm{~min}$

PAM strand

constrained

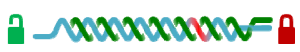

Cas9 -

Cas9+
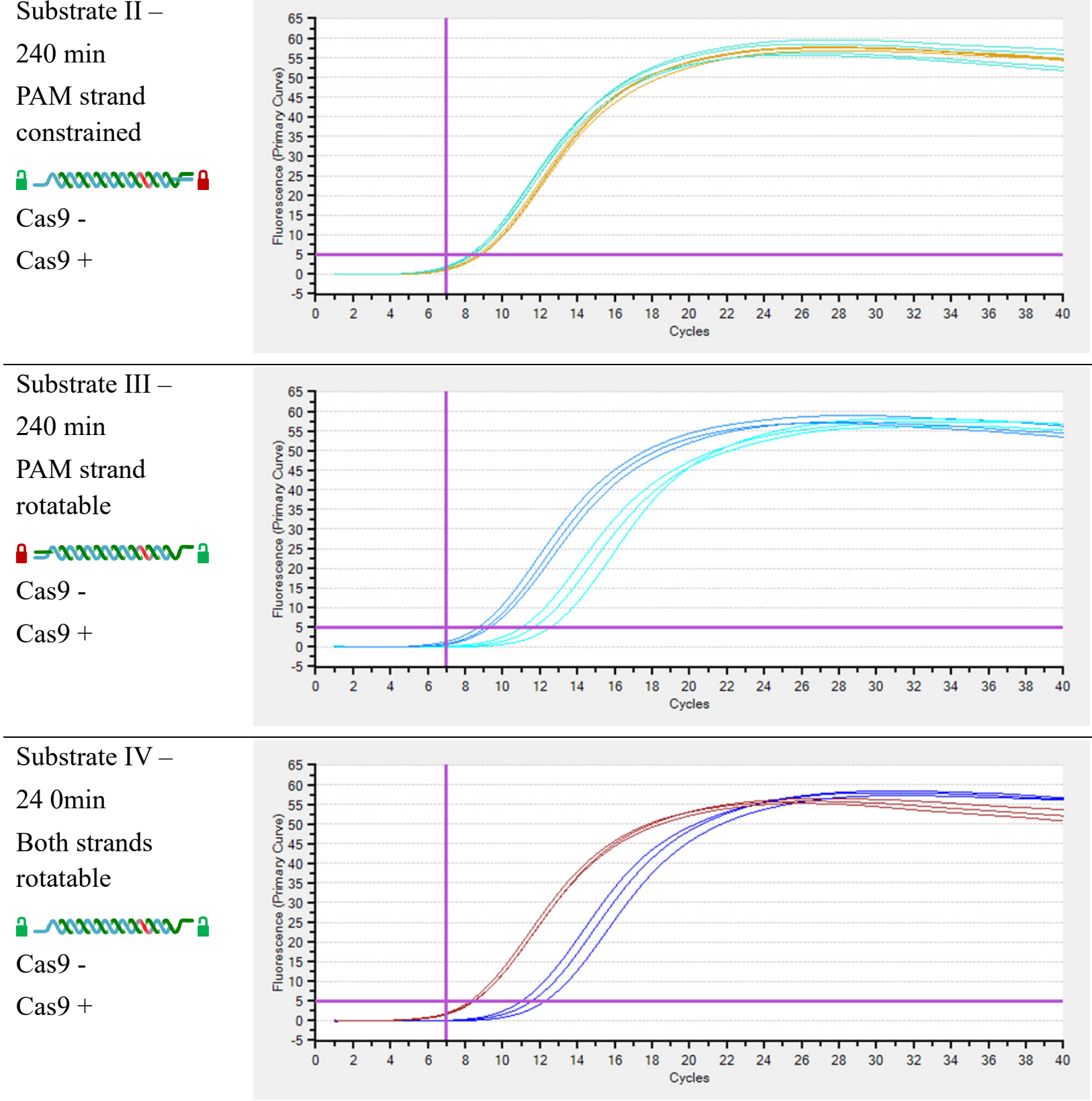
Table S5. Dissociation curves after qPCR reactions with substrate containing Frames I, II, III or IV.

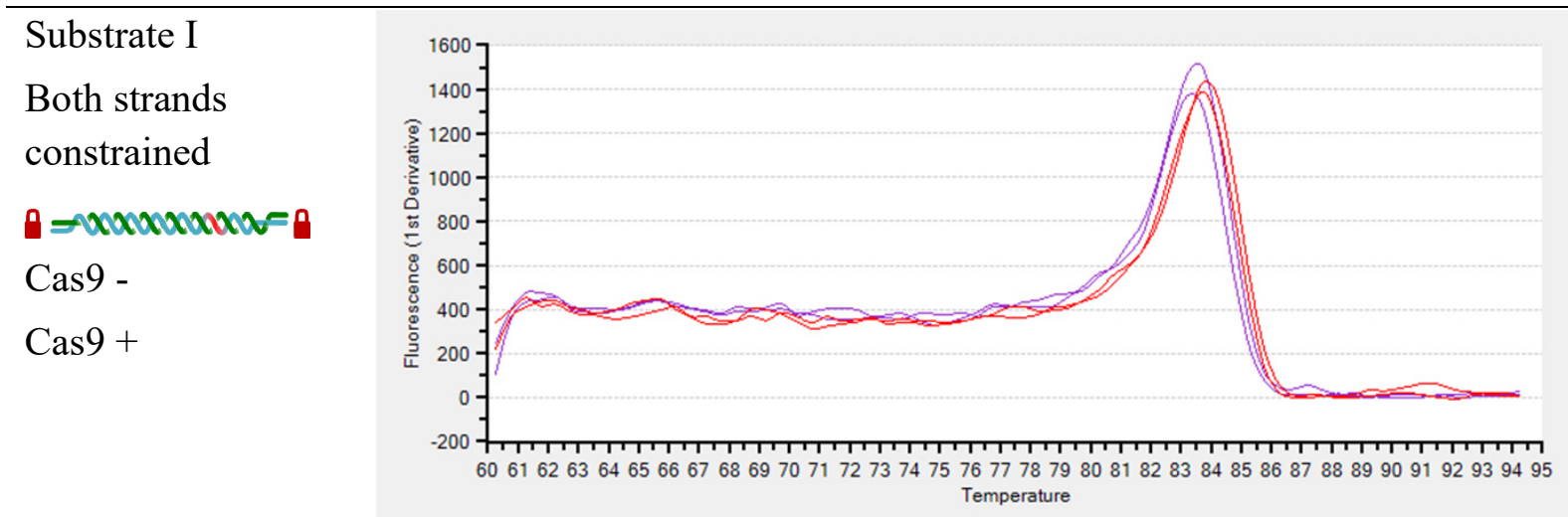

\section{Substrate II}

PAM strand

constrained

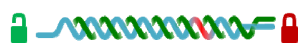

Cas9 -

Cas9+
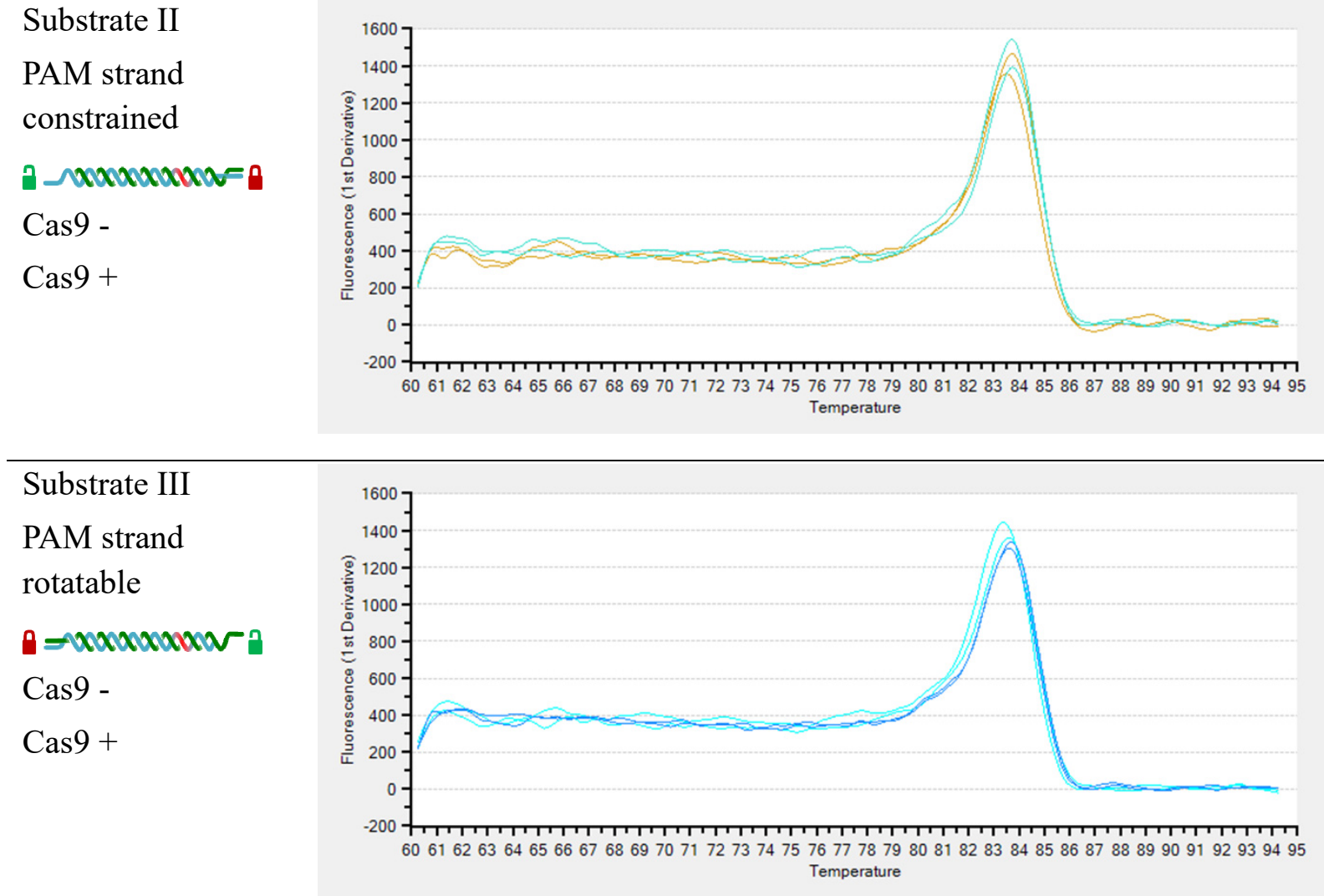

Substrate IV
Both strands
rotatable
Cas9-
Cas9 +

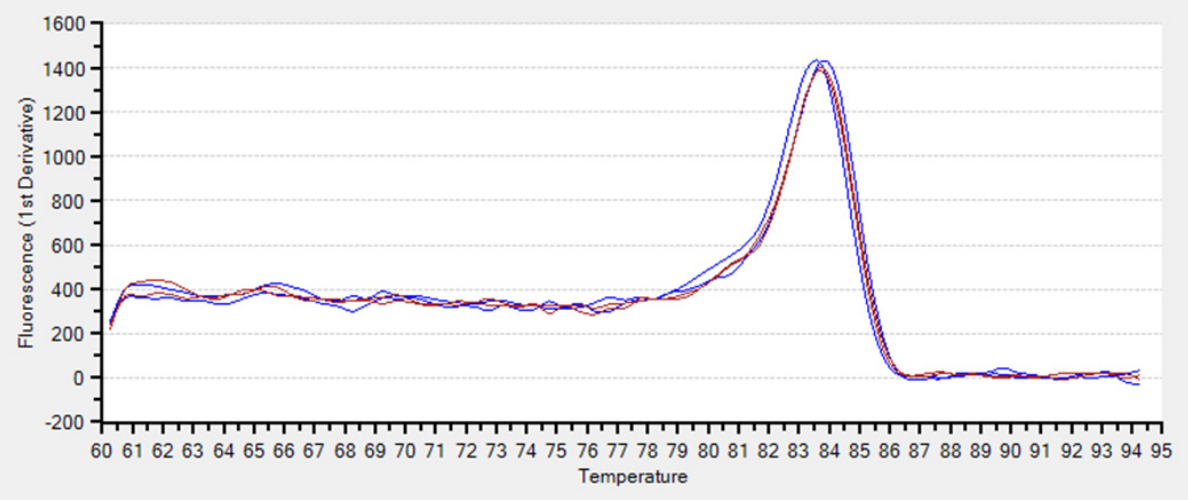


(A) $\begin{array}{llllllllll}M & 1 & 2 & 3 & 4 & 5 & 6 & 7 & 8 & 9\end{array}$
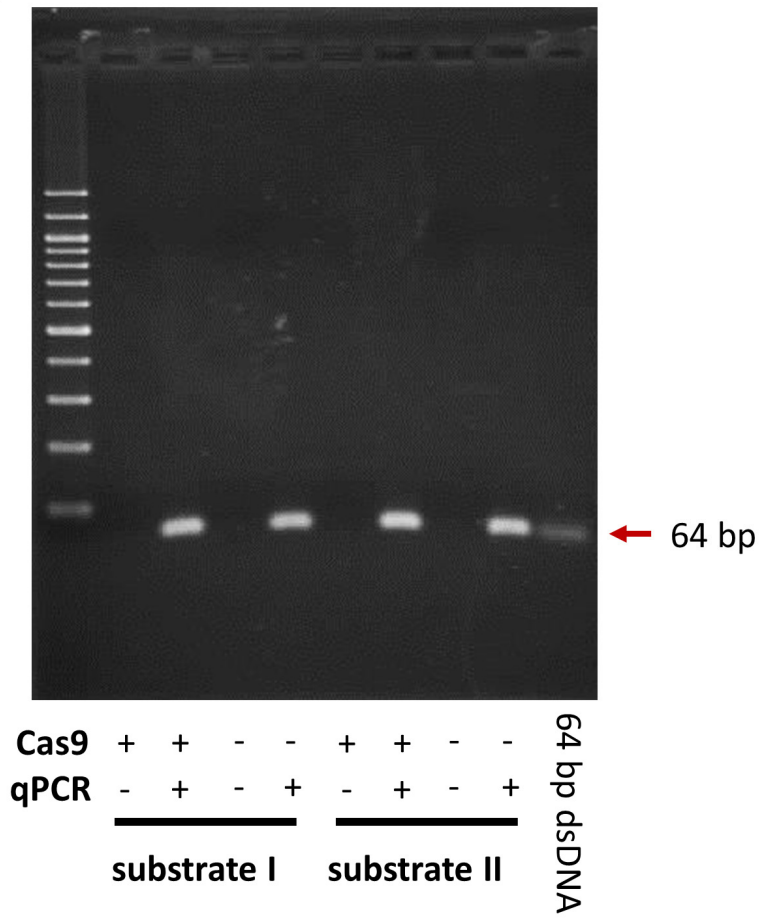

(B) $\quad \begin{array}{llllllllll}M & 1 & 2 & 3 & 4 & 5 & 6 & 7 & 8 & 9\end{array}$

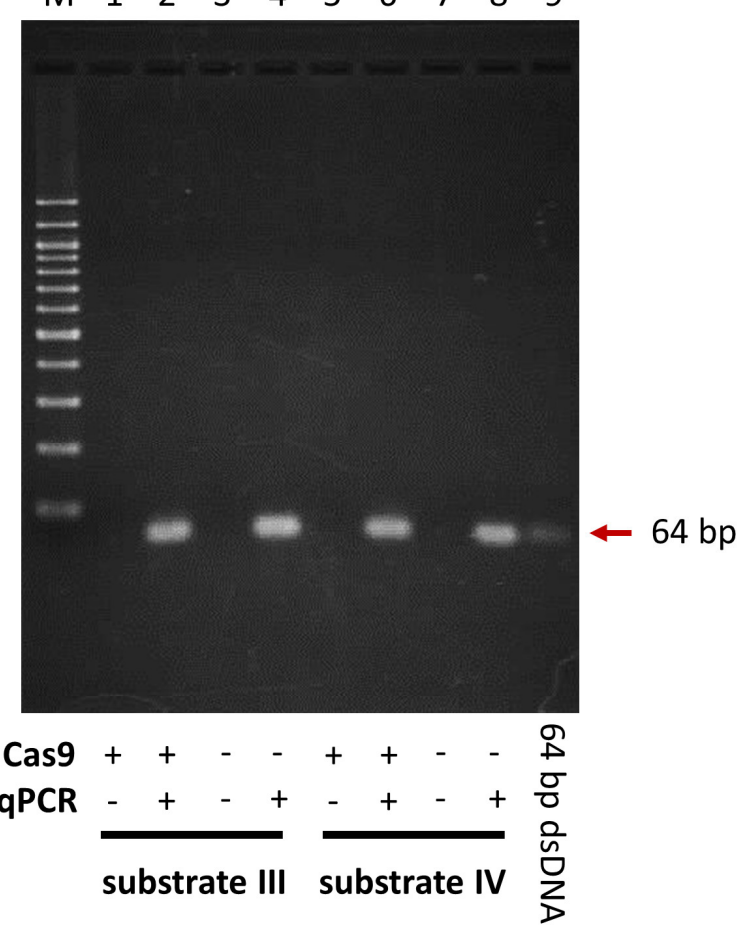

Figure S10. Analysis of reaction products before (-) and after $(+)$ qPCR amplification for reactions with $(+)$ and without (-) Cas9. 2\% Agarose gel, stained with EtBr. (A) M: 100 bp marker; Lanes 1-4: Substrate I; Lanes 5-8: Substrate II; Lane 9: 64 bp marker. Expected amplification product after qPCR: 64 bp. (B) M: 100 bp marker; Lanes 1-4: Substrate III; Lanes 5-8: Substrate IV; Lane 9: 64 bp marker. Expected amplification product after qPCR: $64 \mathrm{bp}$. 


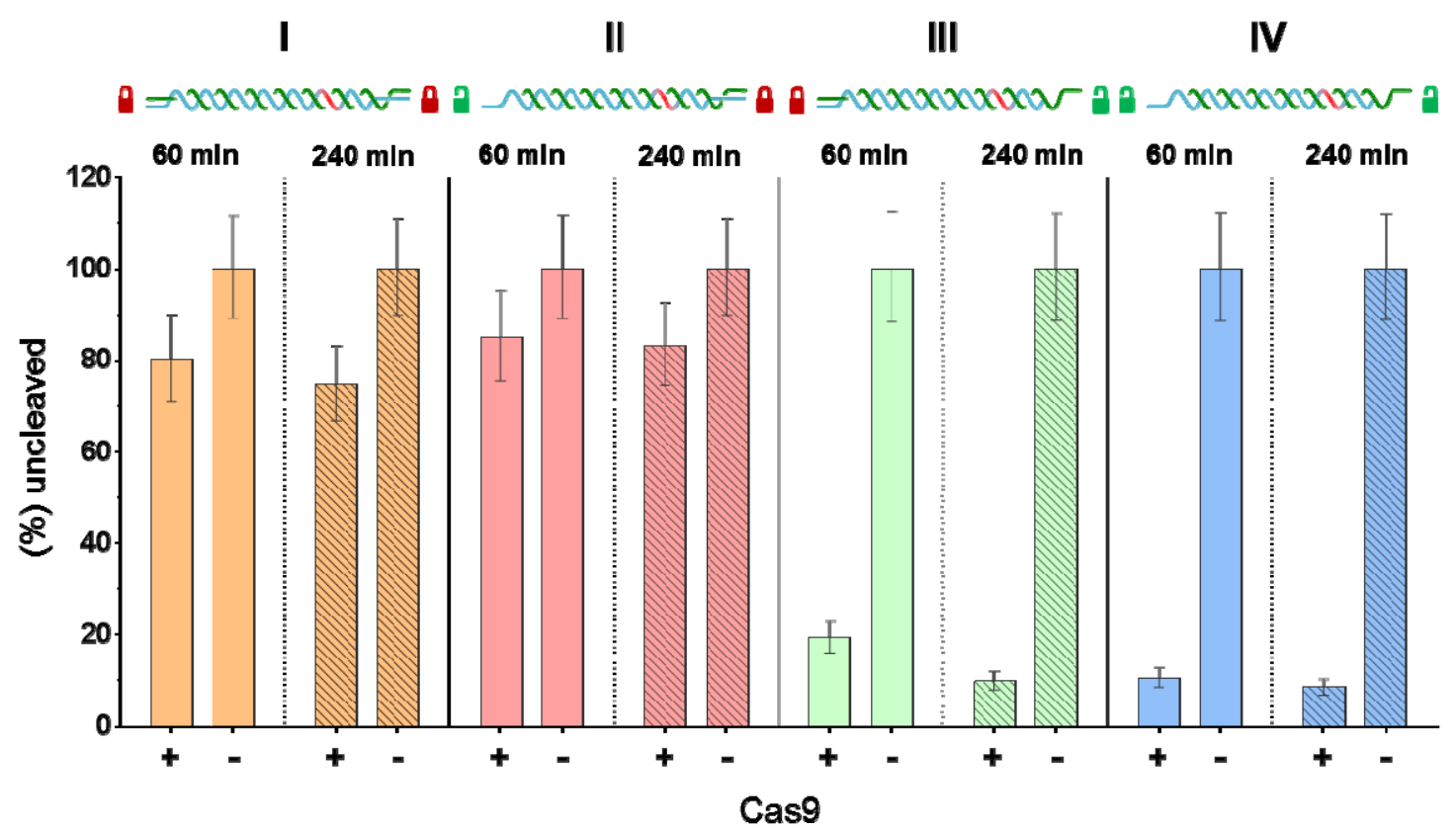

Figure S11. qPCR analysis of Cas9 substrate cleavage. Comparison between $60 \mathrm{~min}$ and $240 \mathrm{~min}$ reaction. Comparison of the quantification of the cleavage of substrates I-IV by Cas9.
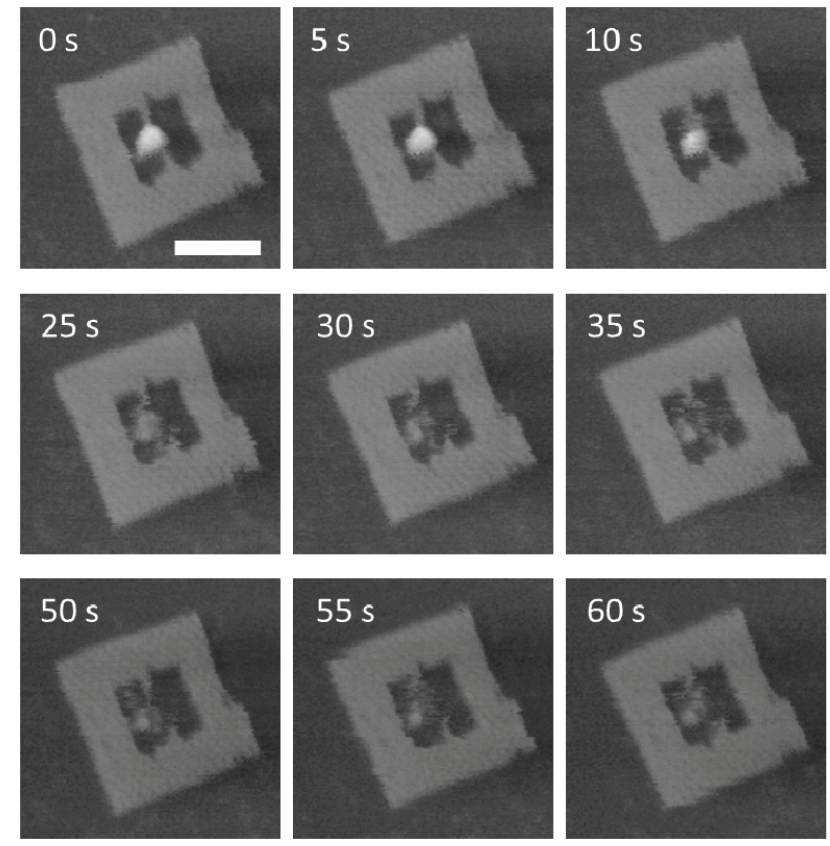
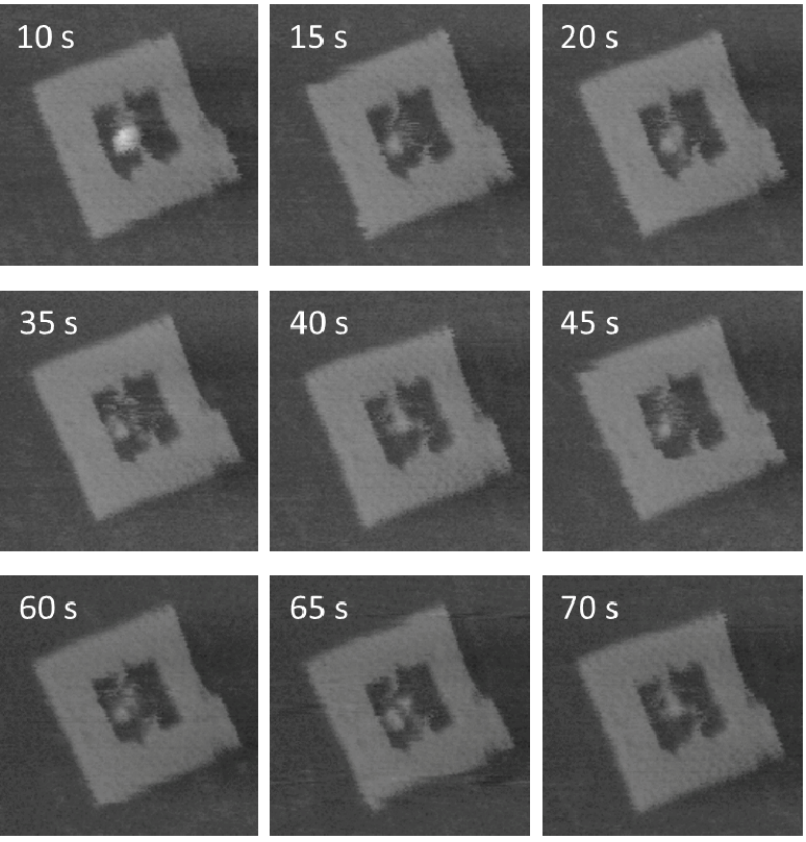

Figure S12. Time-lapse AFM images of the dissociation of Cas9 from the substrate IV containing DNA frame. Scale bar $50 \mathrm{~nm}$. 
Movie S1. High-speed AFM images for the time-lapsed images shown in Figure 4. Images were obtained at room temperature in a buffer containing $10 \mathrm{mM}$ Tris- $\mathrm{HCl}(\mathrm{pH} 7.6), 10 \mathrm{mM} \mathrm{NaCl}, 10 \mathrm{mM}$ $\mathrm{MgCl}_{2}$ and $0.1 \mathrm{mM}$ EDTA. Images were recorded at 0.2 frame/s, play back is at $5 \mathrm{x}(1 \mathrm{frame} / \mathrm{s})$.

Movie S2. High-speed AFM images for the time-lapsed images shown in Figure S12. Images were obtained at room temperature in a buffer containing $10 \mathrm{mM}$ Tris- $\mathrm{HCl}(\mathrm{pH} 7.6), 10 \mathrm{mM} \mathrm{NaCl}, 10 \mathrm{mM}$ $\mathrm{MgCl}_{2}$ and $0.1 \mathrm{mM}$ EDTA. Images were recorded at $0.2 \mathrm{frame} / \mathrm{s}$, play back is at $5 \mathrm{x}(1 \mathrm{frame} / \mathrm{s})$.

\section{Reference}

[1] Y. Suzuki, M. Endo, Y. Katsuda, K. Ou, K. Hidaka, H. Sugiyama, J. Am. Chem. Soc. 2014, 136, 211 218. 